\title{
NOISE REDUCTION IN SPEECH PROCESSING USING IMPROVED ACTIVE NOISE CONTROL (ANC) TECHNIQUE
}

\author{
I. Nancy Catherine ${ }^{1}$, S. Dhandapani ${ }^{2}$ \\ ${ }^{I}$ PG Scholar, ECE Department, Saveetha Engineering College, Tamil Nadu, India \\ ${ }^{2}$ Professor, ECE Department, Saveetha Engineering College, Tamil Nadu, India
}

\begin{abstract}
An improved feed forward adaptive Active Noise Control (ANC) scheme is proposed by using Voice Activity Detector (VAD) and wiener filtering method. The 'speech-plus-noise' periods and 'noise-only' periods are separated using VAD and the unwanted noise is removed by adaptive filtering method. By using Speech Distortion Weighted-Multichannel Wiener Filtering (SDW-MWF) algorithm the noise periods which is present along with the speech signal, is processed and filtered out. The background noise along with the speech samples are removed by the iterative procedure of filtering process. Feed forward based ANC is used to achieve a system with a better noise reduction in speech processing. Adaptive filtering process is carried out and the speech signal without the background noise can be achieved.
\end{abstract}

Key Words: Active Noise Control (ANC), Noise reduction, Adaptive filtering, Feed forward ANC

\section{INTRODUCTION}

Active Noise Control (ANC) also known as noise cancellation is a technique used for reducing the unwanted background noise. It is a method used for reduction in sound by using a power source which is best suited at low frequencies, whereas passive noise control is reduction of noise by using noise isolating materials such as insulation, muffler than a power source. At high frequencies, passive noise control is more effective.

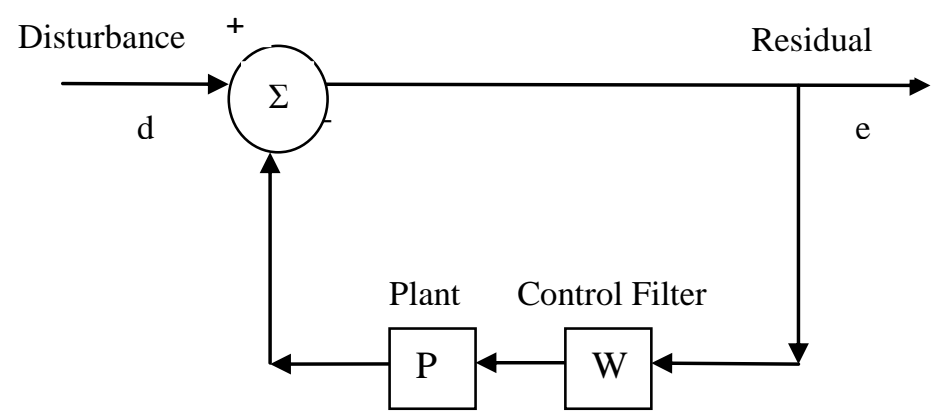

Fig -1: Block Diagram of signal path of an Adaptive ANC system

By introducing a cancelling "antinoise" waveform through an array of secondary sources which are interconnected through an electronic system using a specific signal processing algorithm [5], noise reduction using ANC is achieved.

The FxLMS algorithm is widely used in ANC systems. Due to the presence of an uncorrelated disturbance at the error microphone, the performance of the FxLMS algorithm in steady state will be degraded [1]-[3].

\subsection{Feed forward ANC}

A Feed forward system should be implemented whenever it is possible to obtain a suitable reference signal [8]. The controller and physical system can be separately optimized in this system.

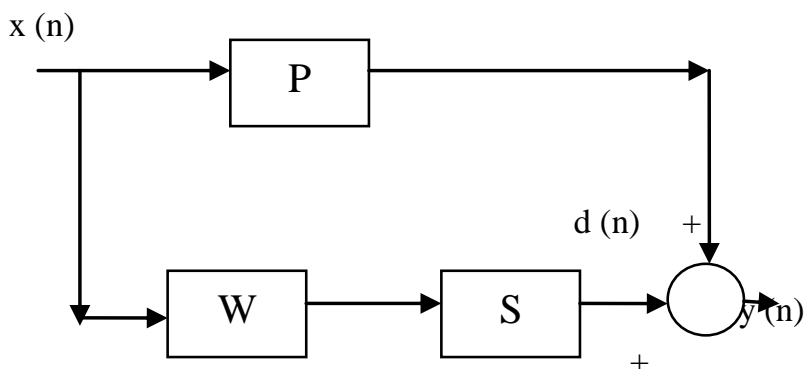

Fig -2: Feedforward ANC

Single-channel feed forward structure has primary noise sensed by reference microphone. Reference signal is used as input to ANC system. Error signal is used to monitor the performance. The goal of this structure is to minimize the error, i.e. the acoustic noise.

\section{PROPOSED METHOD}

Speech signal is given as input which is analog in nature. This analog waveform is converted to digital waveform by sampling and quantization. The speech signal with frequency about $4 \mathrm{KHz}$ is taken as analog input which is converted to discrete signal by means of sampling. Then by the process of quantization, this discrete signal is converted to digital signal. 
An adaptive feed forward based ANC method is proposed which is used to attenuate the multiple noise sources corrupting the speech signal.

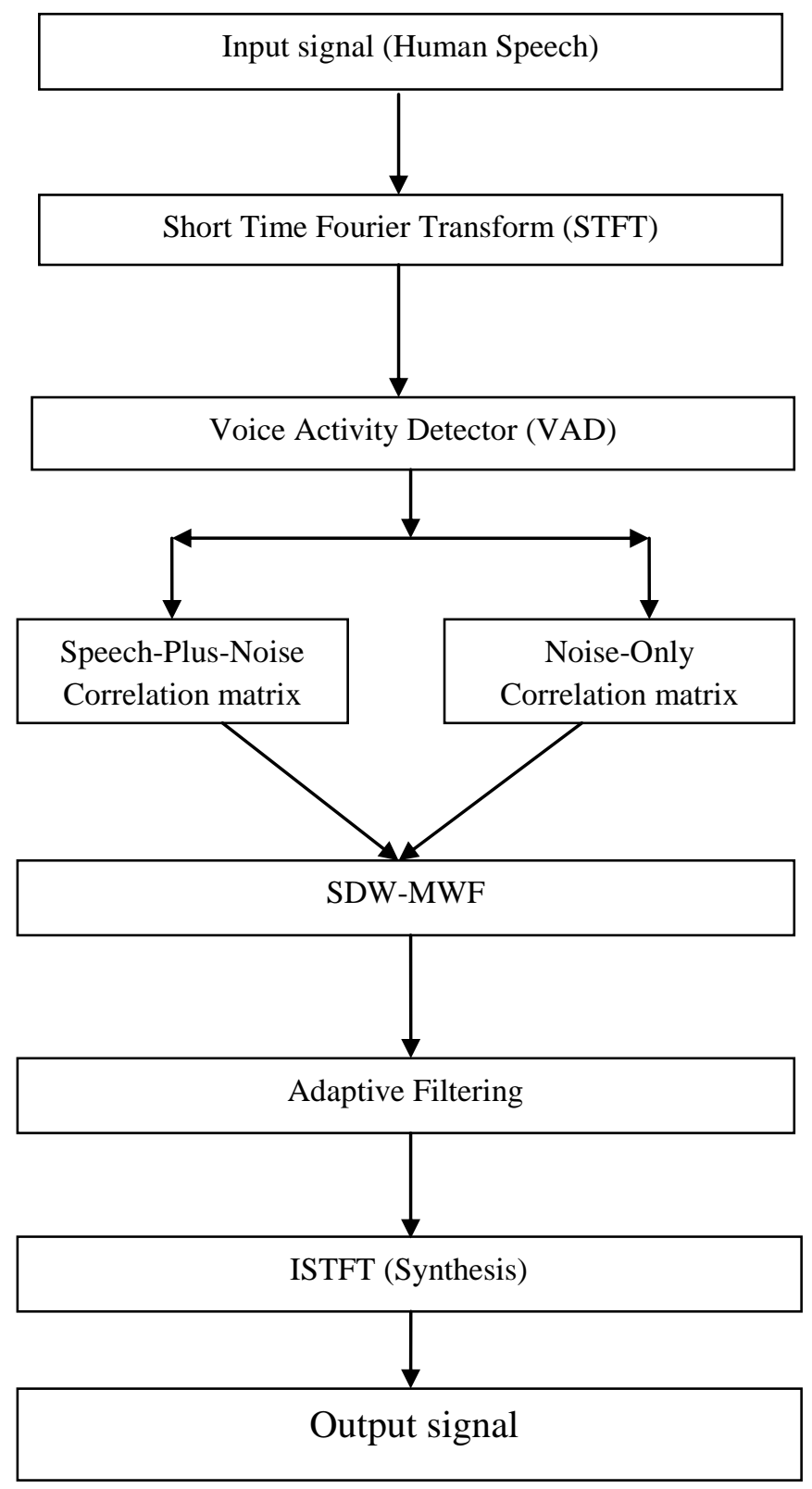

Fig -3: Flow chart of the proposed ANC method

\subsection{Short Time Fourier Transform (STFT)}

By applying Short Time Fourier Transform (STFT) analysis, the digital signal can be segmented into narrow time intervals. Take the Fourier Transform (FT) of each segment. The STFT computed for each window centered at $t=t^{\prime}$ is given below:

$\operatorname{STFT}_{\mathrm{f}}^{\mathrm{u}}\left(\mathrm{t}^{\prime}, \mathrm{u}\right)=\int\left[f(t) \cdot W\left(t-t^{\prime}\right)\right] \cdot \mathrm{e}^{-\mathrm{j} 2 \Pi \mathrm{ut}} \mathrm{dt}$

Where, $\mathrm{t}^{\prime}$ is the time parameter,

$\mathrm{U}$ is the frequency parameter,

$\mathrm{f}(\mathrm{t})$ is the signal to be analyzed

\subsection{Binary VAD}

The input signal consisting of both speeches as well as background noise is processed through the VAD [7]. This is used for improving the efficiency of high speech coding and low-bit rate transmission. VAD firsts counts the number of frames of the sequence using the Zero Crossing Detector (ZCD). It is a technique used to detect the presence or absence of human speech [4].

Based upon the threshold values VAD will separate the speech and noise samples separately. The amplitude of the signal present between this threshold values is detected as speech and the remaining is detected as noise. Now by using adaptive wiener filtering process the unwanted amplitudes of the signal exceeding the maximum and minimum threshold values are eliminated based on the error value calculated. The frame samples are iteratively processed through the filter, so that even the minute noises present along with the speech samples are filtered out. Thus only the speech samples are filtered out. Thus only the speech samples are produced at the output which is of enhanced clarity.

\subsection{Wiener Filter}

Wiener filtering is optimal in terms of the Mean Square Error (MSE). It executes an optimal tradeoff between inverse filtering and noise smoothing. By means of high pass filtering it performs deconvolution by inverse filtering and also removes the noise with a compression operation by low pass filtering.

\subsection{SDW-MWF ${ }_{\mu}$}

The Multi-channel Wiener filter (MWF) is extended by introducing a weighted factor that allows for a trade-off between Noise Reduction (NR) and speech distortion is referred to as the Speech Distortion Weighted Multichannel Wiener Filter $\left(\mathrm{SDW}-\mathrm{MWF}_{\mu}\right)$ [6].

The design criterion of the SDW-MWF ${ }_{\mu}$ is given by

$$
\begin{aligned}
& \mathrm{W}_{\mathrm{MWF} \mu}(\mathrm{k}, \mathrm{l})=\underset{\mathrm{arg} \min }{\operatorname{ar}(\mathrm{k}, \mathrm{l})} \varepsilon\left\{\left|\mathrm{X}_{1}^{\mathrm{s}}(\mathrm{k}, \mathrm{l})-\mathrm{W}^{\mathrm{H}}(\mathrm{k}, \mathrm{l}) \mathrm{X}^{\mathrm{s}}(\mathrm{k}, \mathrm{l})\right|^{2}\right\}+ \\
& \mu \varepsilon\left\{\left|\mathrm{W}^{\mathrm{H}}(\mathrm{k}, \mathrm{l}) \mathrm{X}^{\mathrm{n}}(\mathrm{k}, \mathrm{l})\right|^{2}\right\}
\end{aligned}
$$

And the solution of SDW-MWF is then given by

$$
\mathrm{W}_{\mathrm{MWF \mu}}(\mathrm{k}, \mathrm{l})=\left[\mathrm{R}_{\mathrm{s}}(\mathrm{k}, \mathrm{l})+\mu \mathrm{R}_{\mathrm{n}}(\mathrm{k}, \mathrm{l})\right]^{-1} \mathrm{R}_{\mathrm{s}}(\mathrm{k}, \mathrm{l}) \mathrm{e} 1
$$

\subsection{Adaptive Filtering}

According to an optimization algorithm driven by an error signal, the transfer function is self- adjusted by an adaptive filter.

The difference or error signal e (n) is given by the following formula:

$$
e(n)=d(n)-y(n)
$$




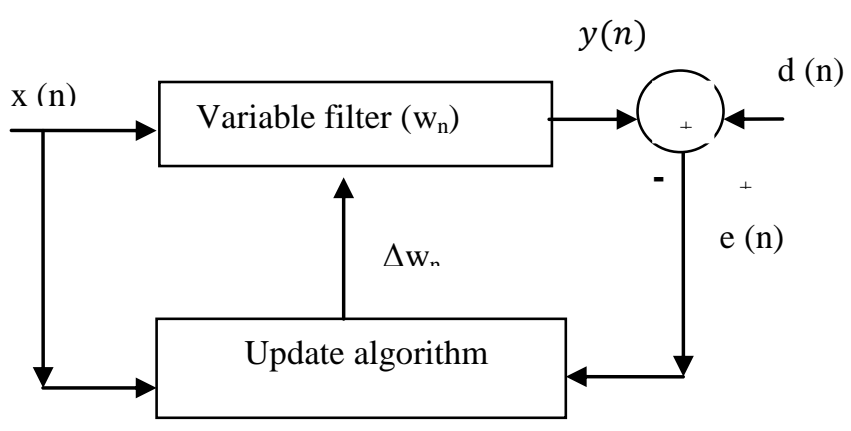

Fig -4: Adaptive Filter

\section{SIMULATION RESULTS}

The experimental setup of the proposed ANC method is designed in MATLAB Simulink. The snapshot of the noise reduction system is shown below:

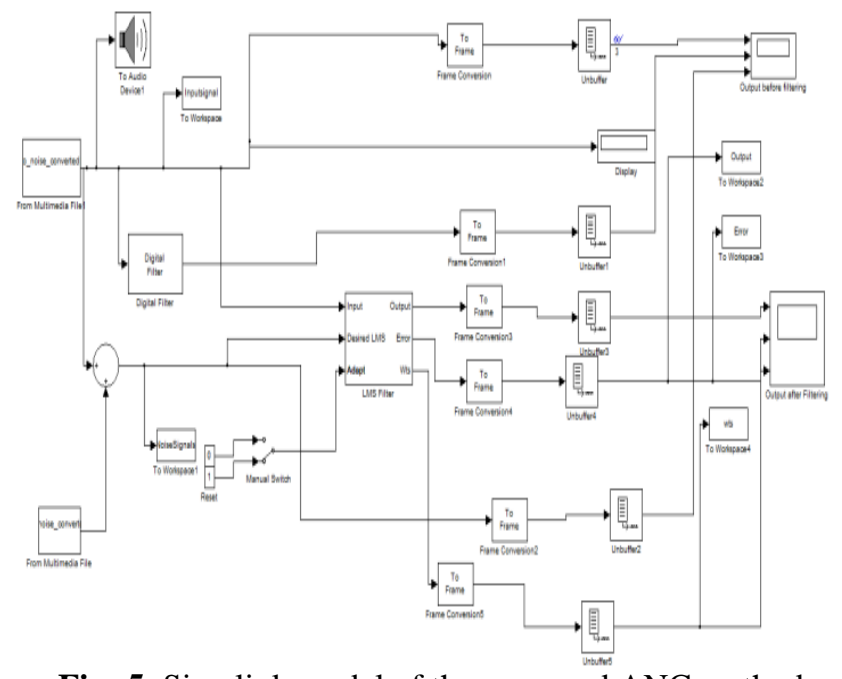

Fig -5: Simulink model of the proposed ANC method

By using VAD and Wiener filtering, the noises from the input signal ('speech plus noise' periods) are removed. Here Adaptive filtering is carried using LMS algorithm and output waveform is shown using scope.

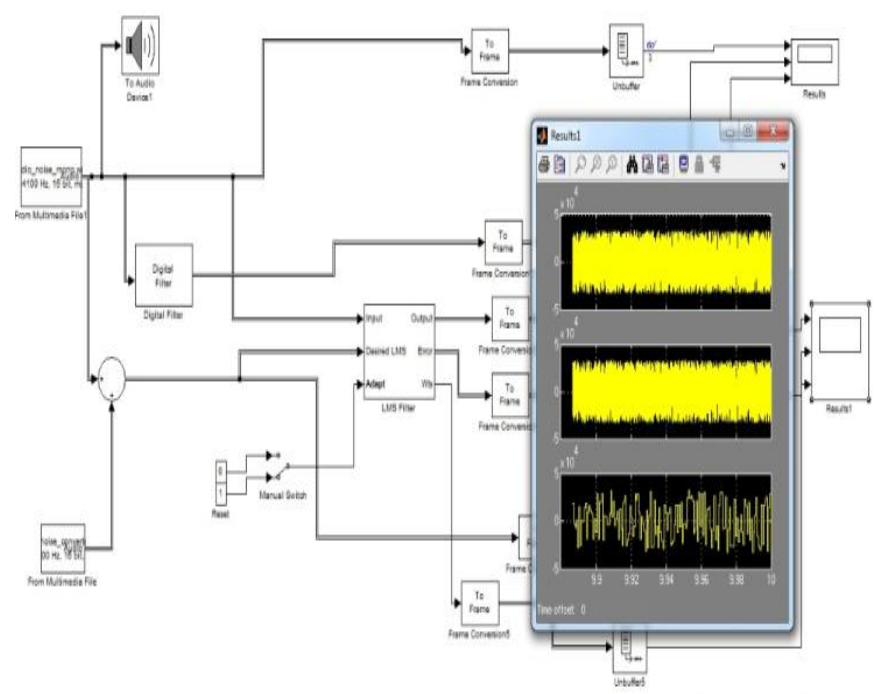

Fig -6: Noise removal using adaptive ANC method
The extraction of only speech periods is done and the output is heard using the speaker. The noise removed output waveform is clearly seen in the snapshot given below:

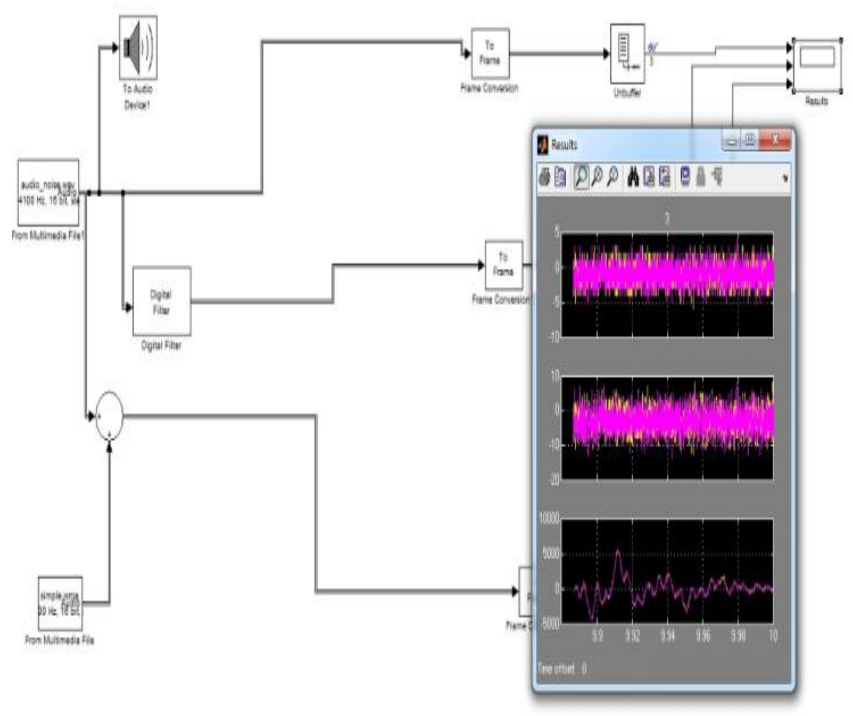

Fig -7: Extraction of speech signal from the input (speech + noise)

\section{CONCLUSION}

In this paper, an adaptive feed forward ANC system is designed using SDW-MWF algorithm. This method is used to attenuate the multiple noise sources scenario and produce clarity at the output. The background noises are reduced by the iterative adaptive filtering method. The designed adaptive feed forward ANC system can be implemented in TMS320C32 for real time applications.

\section{REFERENCES}

[1] Akhtar M.T. and Mitsuhashi W. 'Improving performance of hybrid active noise control systems for uncorrelated narrowband disturbances', IEEE Trans. Audio, Speech, Lang. Process., vol. 19, no. 7, pp. 20582066, 2011.

[2] Chen J. Benesty J. Huang Y. and Doclo S. "New insights into the noise reduction Wiener filter", IEEE Trans. Audio, Speech, Lang. Process., vol. 14, no. 4, pp. 1218-1234, 2006

[3] S. Doclo, A. Spriet, J. Wouters, and M. Moonen, "Frequency-domain criterion for the speech distortion weighted multichannel wiener filter for robust noise reduction", Elsevier Speech Commun., vol. 49, no.7-8, pp. 636-656, 2007.

[4] C. Ganesh Babu and P.T.Vanathi, "Performance analysis of voice activity detection algorithms for robust speech recognition", International Journal of Computing Science and Communication Technologies, vol. 2, no. 1, pp. 288-293, July. 2009.

[5] S. M. Kuo and D. R. Morgan, "Active noise control: A tutorial review", Proc. IEEE, vol. 87, no. 6, pp. 943973, Jun. 1999.

[6] R. Serizel, M. Moonen, J. Wouters and S. H. Jensen, "Integrated active noise control and noise reduction in 
[7] hearing aids", IEEE Trans. Audio, Speech, Lang. Process., vol. 18, no. 6, pp. 1137-1146.

[8] Richard C. Hendriks, Jesper Jensen, and Richard Heusdens, "Noise tracking using DFT domain subspace decompositions", IEEE Trans. Audio, Speech, Lang. Process., vol. 16, no. 3, pp. 541-553, 2008.

[9] Yegui Xiao and Jing Wang "A new feed forward hybrid active noise control system", IEEE Trans. Signal Process., vol. 18, no. 10, pp. 591-594, 2011.

\section{BIOGRAPHIES}

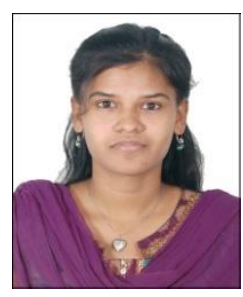

I. Nancy Catherine received the B.E. degree in Electronics and Communication Engineering in the year 2011 and pursuing M.E. degree in VLSI Design in Saveetha Engineering College. Her area of interest include Speech Signal processing and VLSI Design.

Dr. S. Dhandapani received the B.E. degree in Electrical and Electronics Engineering and M.E. degree in Applied Electronics from Bharathiar university in 1998 and 2003 respectively and $\mathrm{Ph} . \mathrm{D}$. degree in Information and Communication Engineering from Anna university, Chennai in 2012. Currently, he is a Professor in the Department of Electronics and Communication Engineering, Saveetha Engineering College, Chennai. $\mathrm{He}$ is a life member of ISTE, IETE and IAENG. He has published more than 10 research papers. His research interests include digital image processing, network security and VLSI design. 